\title{
Geometrical frustration: A study of four-dimensional hard spheres
}

\author{
J. A. van Meel \\ FOM Institute for Atomic and Molecular Physics, Kruislaan 407, 1098 SJ Amsterdam, The Netherlands \\ D. Frenkel \\ Department of Chemistry, University of Cambridge, Lensfield Road, Cambridge CB2 1EW, United Kingdom \\ and FOM Institute for Atomic and Molecular Physics, Kruislaan 407, 1098 SJ Amsterdam, The Netherlands \\ P. Charbonneau \\ Department of Chemistry, Duke University, Durham, North Carolina 27708, USA
}

(Received 26 November 2008; published 3 March 2009)

\begin{abstract}
The smallest maximum-kissing-number Voronoi polyhedron of three-dimensional (3D) Euclidean spheres is the icosahedron, and the tetrahedron is the smallest volume that can show up in Delaunay tessellation. No periodic lattice is consistent with either, and hence these dense packings are geometrically frustrated. Because icosahedra can be assembled from almost perfect tetrahedra, the terms "icosahedral" and "polytetrahedral" packing are often used interchangeably, which leaves the true origin of geometric frustration unclear. Here we report a computational study of freezing of 4D Euclidean hard spheres, where the densest Voronoi cluster is compatible with the symmetry of the densest crystal, while polytetrahedral order is not. We observe that, under otherwise comparable conditions, crystal nucleation in four dimensions is less facile than in three dimensions, which is consistent with earlier observations [M. Skoge et al., Phys. Rev. E 74, 041127 (2006)]. We conclude that it is the geometrical frustration of polytetrahedral structures that inhibits crystallization.
\end{abstract}

DOI: 10.1103/PhysRevE.79.030201 PACS number(s): 61.20.-p, 64.70.dm, 64.60.qe, 64.30. $-\mathrm{t}$

Most glasses form under conditions where the thermodynamically stable state of the system is crystalline. Good glass formers should therefore be poor crystallizers. Geometrical frustration is one of the factors that may prevent the formation of the ordered phase and therefore help physical glass formation [1]. There is also evidence that such frustration increases the height of the crystallization nucleation barrier of liquid metals [2]. Isotropic simple liquids are often considered frustrated because the fivefold symmetry of the liquid icosahedra cannot pack as a regular lattice. This scenario contrasts with what happens in a fluid of two-dimensional (2D) disks, where hexagonal order is both locally and globally preferred and where crystallization is particularly easy.

Several physical mechanisms have been proposed to support the formation of icosahedra. On the one hand, Frank, considering the optimal way for kissing spheres to cluster around a central one, found the icosahedron to be more stable than the cubic-lattice unit cells for the Lennard-Jones model [3]. Although the original argument relies on the energetics of spurious surface effects [4], mean-field studies correcting for solvation leave the result unchanged $[5,6]$. The icosahedron, the smallest maximum kissing-number Voronoi polyhedron, is optimally packed. It offers the most free volume to surface spheres, so it is also preferred entropically. On the other hand, the polytetrahedral scenario ascribes the presence of icosahedra to their facile assembly from quasiperfect tetrahedra, themselves the smallest Delaunay decomposition of space $[7,8]$. But is it the packing of Voronoi polyhedra or the packing of Delaunay hypertriangles that counts? Experiments [9-11] and simulations [12,13] only manage to identify icosahedral order in limited quantities, even in deeply supercooled systems. Recent studies indicate that liquid polytetrahedral order is a lot more varied $[14,15]$ than the icosahedral picture suggests. Yet because of the geometrical ambiguity, the equation of the icosahedron with frustration is difficult to assess.

Looking at crystallization in a system where polytetrahedral frustration does not correspond to a symmetric closedshell structure like the icosahedron would help. Precisely such an example is provided by the freezing of 4D spheres that we study in this Rapid Communication. It is, of course, somewhat unsatisfactory to perform a numerical study of a system that cannot be probed experimentally. However, there are other examples (e.g., renormalization-group theory) where higher-dimensional model systems serve as a very useful reference state for the theoretical description of our $3 \mathrm{D}$ world. The objective of the numerical study that we report here is therefore not to present quantitative estimates of crystal nucleation barriers in four dimensions (even though we obtain these numbers too), but to shed more light on the nature and role of geometrical frustration and the ease of crystallization.

The $D_{4}$ crystal phase is formed by stacking, without voids, 24-cell Platonic polytopes [16,17]. In general, $D_{d}$ lattices are obtained by inserting an additional sphere in each void of a $d$-dimensional cubic lattice. In three dimensions the spacing between the spheres on the original cubic lattice increases to form a body-centered-cubic crystal; in four dimensions the additional sphere fits perfectly in the hole and leads to a unique, high-symmetry crystal with a volume fraction $\eta=\pi^{2} / 16 \approx 0.617$, which is postulated to be maximal [19]. There exist other dense 4D lattices, such as $A_{4}$ and $A_{4}^{*}$, but $D_{4}$ packs over $10 \%$ more densely and offers more nearestneighbor contacts. $D_{4}$ 's unit cell, the 24-cell, is made of 24 octahedral cells and is a Platonic polytope that has no analog in other dimensions [17]. Placing 24 kissing spheres around a central one in the 24-cell arrangement is the densest closedshell cluster of 4D spheres [18] and is postulated to be 
unique [19]. Even accounting for solvation effects, clusters with the 24-cell geometry are locally preferred. Unlike in three dimensions, for an equal number of particles 4D polytetrahedral clusters do not form more interparticle contacts than the 24-cell, and their slightly larger radius offers less, not more stabilization [5]. The symmetry match between the 24-cell and the $D_{4}$ lattice therefore guarantees that no frustration arises from maximally kissing clusters. But neither the 24-cell nor any other unit cell can be assembled from (nearly) regular 4D tetrahedra. Four-dimensional spheres are thus an ideal system to clarify the origin of geometrical frustration. An earlier compaction study of 4D spheres found that spontaneous crystallization is suppressed with increasing dimension and concluded that geometrical frustration is likely to increase with dimension [20]. However, this work could not disentangle the different contributing factors, because such an analysis requires knowledge of the equilibrium phase diagram, of the dynamical properties of the fluid phase, and of the crystal nucleation barriers. Our computational study addresses these questions. To this end, we first locate the 4D freezing transition, quantify the fluid order, and then compute the free-energy barrier to nucleation at different supersaturations.

Interestingly, although the equations of state of both the fluid and the crystal phases of 4D hard spheres were computed in the early 1980s [23], we are not aware of any numerical determinations of the solid-fluid coexistence point. Using a quasi-Maxwell construction [24] at the crystal stability limit [20], we can use these results to approximate the coexistence range $\eta_{\text {coex }}=0.29-0.35$, but this is insufficiently accurate. To the best of our knowledge, density functional theory has only been applied to the fluid- $A_{4}$ coexistence [25]. In order to precisely locate the freezing point, we thus performed standard NVT Monte Carlo (MC) simulations to compute the equation of state of hard spheres outside the range studied in Ref. [23]. As a test, we also performed constant NPT simulations and verified that the two techniques yielded consistent results. In what follows, we use the particle diameter $\sigma$ as our unit of length and the thermal energy $k_{B} T$ as our unit of energy. The equation of state of 4D spheres is related to the value of the pair-distribution function $g(r)$ at contact, $P v_{0} / \eta=1+8 \eta g\left(1^{+}\right)$, where $v_{0}$ is the volume of a $4 \mathrm{D}$ sphere and $g\left(1^{+}\right)$is the value of the radial distribution function at contact [21]. The results for the fluid and two crystal phases are presented in Fig. 1 for systems containing $2048\left(D_{4}\right)$ and 4096 (fluid and $\left.A_{4}\right)$ particles. The equation of state could not be calculated for $A_{4}^{*}$, because it is mechanically unstable, which makes it unlikely to contribute to the crystallization process. We will not consider it further. To locate the fluid-solid coexistence regime, we need to determine the absolute free energy of the solid at least at one point [26]. The absolute Helmholtz free energy per particle $f$ of the $D_{4}$ and $A_{4}$ crystals at $\eta=0.37$ is obtained by the Einstein crystal method [27]. The free energy at other densities can then be obtained by thermodynamic integration. We find $D_{4}$ to be the thermodynamically stable crystal phase. The fluid- $D_{4}$ coexistence pressure $P_{\text {coex }}$ (Fig. 1, lower inset) allows one to read off the melting and freezing densities by common tangent construction (Fig. 1, upper inset). The resulting two-phase region $\eta_{\text {coex }}=0.288-0.337$ is compatible

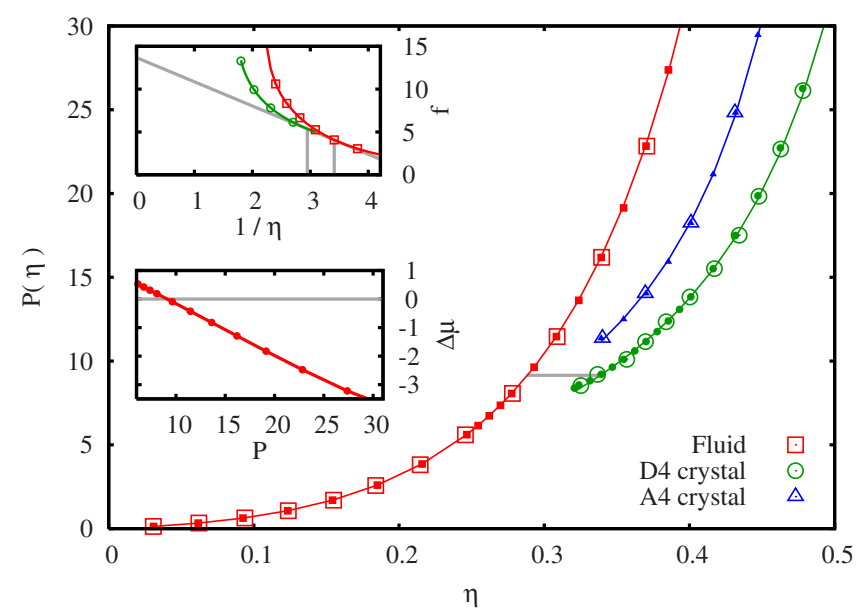

FIG. 1. (Color online) Equations of state of 4D hard spheres at constant $V$ (solid symbols) and $P$ (open symbols) extend earlier molecular dynamics results [23]. The Padé approximant for the fluid [21] and Speedy fits for the crystals [22] are given for reference (lines). Bottom inset: at coexistence, chemical potentials are equal; thus, $P_{\text {coex }}=9.15$ and $\mu_{\text {coex }}=13.68$. Top inset: the common tangent to the free-energy curves pinpoints the phase transition boundaries: $\eta_{\text {freeze }}=0.288$ and $\eta_{\text {melt }}=0.337$.

with the rough estimate above. The thermodynamic driving force for crystallization in the supersaturated fluid at constant pressure is the difference in chemical potential, $\Delta \mu=\mu_{D_{4}}$ $-\mu_{\text {fluid }}$, between the two phases displayed in the lower inset of Fig. 1.

To characterize the structure of the fluid and identify the formation of crystallites, we need a local criterion that distinguishes crystal from fluid. Studies in two and three dimensions suggest that order parameters derived from invariant combinations of spherical harmonics $Y_{l}$ of degree $l$ might suffice $[28,29]$. In high dimensions, it is more convenient to rewrite the second-order invariant in terms of Gegenbauer polynomials $G_{l}^{n}$, where $n=d / 2-1$, using the sum rule [19]. The $(l+1)^{2} 4 \mathrm{D}$ spherical harmonics give

$$
G_{l}^{1}\left(\hat{\mathbf{r}}_{1} \cdot \hat{\mathbf{r}}_{2}\right)=\frac{2 \pi^{2}}{(l+1)^{2}} \sum_{m=1}^{(l+1)^{2}} Y_{l}^{m}\left(\hat{\mathbf{r}}_{1}\right) \overline{Y_{l}^{m}\left(\hat{\mathbf{r}}_{2}\right)},
$$

where $\hat{\mathbf{r}}_{i}$ are unit vectors. The local order correlator is

$$
q_{l}^{i, j}=\mathbf{q}_{l}(i) \cdot \mathbf{q}_{l}(j)=\frac{1}{N(i) N(j)} \sum_{\alpha=1}^{N(i)} \sum_{\beta=1}^{N(j)} G_{l}^{1}\left(\hat{\mathbf{r}}_{\alpha i} \cdot \hat{\mathbf{r}}_{\beta j}\right),
$$

where the indices $\alpha$ and $\beta$ run over the number of neighbors contained within a distance equal to the first minimum of $g(r)$. The local order correlation distinguishes between different geometrical environments: $q_{6}$ sets apart fluidlike particles from those within a $D_{4}$ or an $A_{4}$ lattice, while $q_{4}$ discriminates between the two crystals (Fig. 2).

As freezing in four dimensions is a first-order phase transition, we expect crystallization to proceed via nucleation and growth. A Landau free-energy analysis predicts that crystals with reciprocal lattice vectors forming equilateral triangles should initiate the nucleation [30]. Though this argu- 


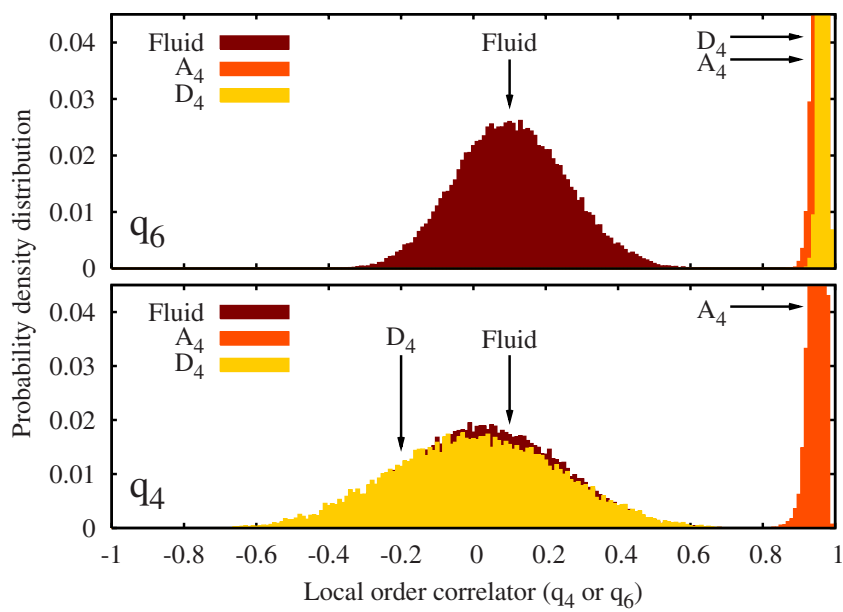

FIG. 2. (Color online) Distribution of the local order correlator with $l=4$ (top) and $l=6$ (bottom) at $P=19$.

ment has met only limited success in three dimensions [29], in four dimensions it supports the preferential nucleation of $D_{4}$, in line with the thermodynamic drive. To estimate the ease of crystallization, we compute the free-energy barrier for crystal nucleation $\Delta G^{*}$. Classical nucleation theory (CNT) [31] derives from the thermodynamic drive $\Delta \mu$ and the interfacial free energy $\gamma$ of a spherical crystallite a freeenergy functional that depends on the size $n$ of the crystallite:

$$
\Delta G(n)=S_{d}\left(n / \rho_{x}\right)^{(d-1) / d} \gamma-n \Delta \mu,
$$

where $\rho_{x}$ is the crystal density at a given pressure and the shape-dependent prefactor is $S_{4}=\left(128 \pi^{2}\right)^{1 / 4}$ for $4 \mathrm{D}$ spheres. The resulting maximal barrier height is then

$$
\Delta G^{*}\left(n^{*}\right)=\frac{27 \pi^{2} \gamma^{4}}{2 \rho_{D_{4}}^{3} \Delta \mu^{3}}
$$

at the critical cluster size $n^{*}$. The rate of nucleation per unit volume $I$ is given by $I=\kappa \exp \left(-\Delta G^{*}\right)$, where $\kappa$ is a kinetic prefactor that is proportional to the diffusion coefficient in the fluid phase [29]. Although schematic, this level of theory is sufficient to clarify the contribution of geometrical frustration through an analysis of $\gamma$. Within the CNT framework the geometrical mismatch in three dimensions between icosahedral and crystal order should lead to a relatively large $\gamma$, while in four dimensions one might expect $\gamma$ to be small if the locally preferred cluster scenario is valid, but not for polytetrahedral frustration.

Results for 3D crystallization are available [29], so only a few 4D barriers are needed to complete the picture. Crystallization being a rare event in this regime, we perform constant-pressure MC runs with umbrella sampling to bias the growth of a crystal cluster from the fluid [26]. A standard algorithm is employed to identify the crystallites $[28,29]$. We link pairs of nearest neighbors with $q_{6}>0.4$. If a particle has more than five links, it is deemed crystalline. The number of particles in the largest crystallite is then the order parameter. The resulting free-energy profiles are presented in Fig. 3. Although $q_{6}$ does not discriminate between $A_{4}$ and $D_{4}$ crys-

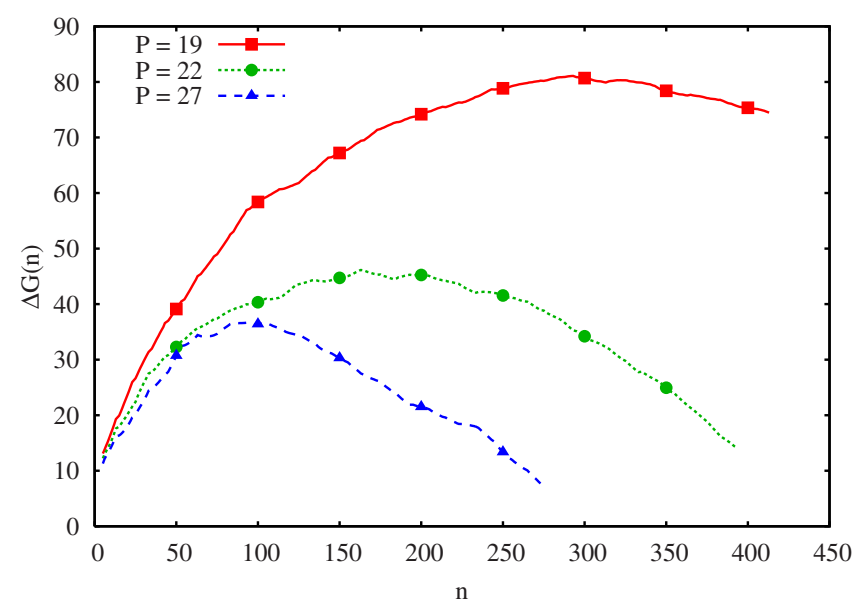

FIG. 3. (Color online) Free-energy barriers for 4096 particles at various supersaturations.

tals, further checks with $q_{4}$ show that only the latter nucleates. In four dimensions a low- $q_{6}$ cutoff value is required, because of the minimal overlap between the crystalline and fluid regions (Fig. 2), and consequently, noncompact clusters are initially observed. Although the clusters irreversibly compactify, the process can be very slow. To reduce the computational burden, the system is first equilibrated by growing the total number of links in the largest crystallite. The low- $q_{6}$ cutoff also artificially inflates the measured critical cluster size. A fit to the CNT functional form [Eq. (3)] is thus of little use in extracting $\gamma$. However, because the barrier height is unaffected by this biasing choice and $\Delta \mu$ is known, we can obtain $\gamma$ from Eq. (4) directly. To validate the implied size of the CNT critical cluster $n_{\mathrm{CNT}}^{*}$, we compare it to the cluster size obtained by imposing a purely crystalline linking criterion $q_{6}>0.65$ to the configurations at the top of the barrier. The difference between the two (Table I) is no more than $25 \%$, which is remarkably good in this context.

The results of Fig. 3 allow us to conclude that the very slow crystallization of 4D spheres observed in the study of Ref. [20] is due to the presence of a considerably higher 4D nucleation barrier than at the same supersaturation in three dimensions. Slow nucleation could also be due to a low value of the kinetic prefactor $\kappa$, which would require that the diffusion of particles in the dense fluid be anomalously slow. But simulations with the code of Ref. [20] show no evidence for slow diffusion, not even at the highest pressures studied. The slow crystallization is thus consistent with a high degree of geometrical frustration in 4D fluids. Based on the similarity between the number of neighbors within the first peak of

TABLE I. Simulation parameters for Fig. 3, along with the corresponding CNT parameters. The critical cluster size $n^{*}$ is obtained using a stricter order parameter (see text).

\begin{tabular}{lccccc}
\hline \hline$P$ & $\Delta \mu$ & $\Delta G^{*}$ & $\gamma_{\mathrm{CNT}}$ & $n^{*}$ & $n_{\mathrm{CNT}}^{*}$ \\
\hline 19 & -1.8 & 81 & 1.80 & 157 & 133 \\
22 & -2.3 & 42 & 1.94 & 75 & 60 \\
27 & -3.2 & 37 & 2.4 & 40 & 35 \\
\hline \hline
\end{tabular}


$g(r)$ and the maximal kissing number, Skoge et al. speculated that high-dimensional fluids contain a number of deformed crystalline unit cells, rather than polytetrahedral structures [20]. However, the clear difference between the fluid and the 24-cell shown by the local order correlator (Fig. 2) suggests this not to be the case. The similarity between the kissing number and the number of first neighbors can instead be explained by a wide first peak of $g(r)$ (not shown) that accommodates nonkissing neighbors in polytetrahedral clusters. Because the "locally preferred" 24-cell has little to do with geometrical frustration, our results support the generic polytetrahedral structures as the source of frustration. By dimensional analogy, we infer that the "locally preferred" icosahedron is not singular, but instead one of the many possible geometrically frustrating structures, and explains its limited presence in fluids. The dimensionless surface freeenergy density $\gamma \sigma^{d-1} /\left(k_{B} T\right)$ is at least 2-3 times larger in four dimensions than in three dimensions, which indicates that geometrical frustration is surprisingly rather weak in three dimensions. It is this weakness that helps make hardsphere crystallization so prevalent. The interesting puzzle is therefore not to identify the origin of 3D frustration, but the source of its mildness. One possibility is that the tetrahedra that are part of the fcc structure (none are found in $D_{4}$ ) relax the geometrical frustration and therefore reduce the interfacial tension. Another possibility is that the "planetary pertur- bations" that allow an exchange of the positions of spheres at the surface of an icosahedron by sliding go through a cuboctahedron configuration, which is the fcc unit cell [17]. If common, this phenomenon would imply that not all polytetrahedral structures are equally frustrating and that icosahedra might in fact be early nucleation sites.

The large values for the height of the nucleation barrier of $4 \mathrm{D}$ crystals, as well as the evidence (Fig. 2) that the local structures in the fluid and the $D_{4}$ crystal are rather different, indicate that it is the Delaunay packing that matters. This finding underlines that one should be rather careful in caricaturing the nature of frustration as icosahedral in 3D liquids. Icosahedra are but one of the many possible polytetrahedral arrangements, and little indicates that it plays a more prominent role in geometrical frustration than others. Note that the difficulty to crystallize 4D spheres makes them, as well as their higher-dimensional equivalents, promising testing grounds for theories of packing and glass-forming liquids.

We thank B. Charbonneau, B. Mulder, S. Abeln, G. Tarjus, and R. Mossery for their help at various stages of this project. The work of the FOM Institute is part of the research program of FOM and is made possible by financial support from the Netherlands Organization for Scientific Research (NWO).
[1] G. Tarjus, S. A. Kivelson, Z. Nussinov, and P. Viot, J. Phys.: Condens. Matter 17, R1143 (2005).

[2] T. Schenk, D. Holland-Moritz, V. Simonet, R. Bellissent, and D. M. Herlach, Phys. Rev. Lett. 89, 075507 (2002).

[3] F. C. Frank, Proc. R. Soc. London, Ser. A 215, 43 (1952).

[4] J. P. K. Doye and D. J. Wales, J. Phys. B 29, 4859 (1996).

[5] S. Mossa and G. Tarjus, J. Chem. Phys. 119, 8069 (2003).

[6] S. Mossa and G. Tarjus, J. Non-Cryst. Solids 352, 4847 (2006).

[7] D. R. Nelson, Defects and Geometry in Condensed Matter Physics (Cambridge University Press, New York, 2002).

[8] J.-F. Sadoc and R. Mosseri, Geometrical Frustration (Cambridge University Press, Cambridge, England, 1999).

[9] A. Di Cicco, A. Trapananti, S. Faggioni, and A. Filipponi, Phys. Rev. Lett. 91, 135505 (2003).

[10] A. Di Cicco and A. Trapananti, J. Non-Cryst. Solids 353, 3671 (2007).

[11] T. Aste, M. Saadatfar, and T. J. Senden, Phys. Rev. E 71, 061302 (2005).

[12] T. Kondo and K. Tsumuraya, J. Chem. Phys. 94, 8220 (1991).

[13] N. Jakse and A. Pasturel, Phys. Rev. Lett. 91, 195501 (2003).

[14] A. V. Anikeenko and N. N. Medvedev, Phys. Rev. Lett. 98, 235504 (2007).

[15] A. V. Anikeenko, N. N. Medvedev, and T. Aste, Phys. Rev. E 77, 031101 (2008).

[16] H. S. M. Coxeter, Regular Polytopes (Dover, New York,
1973).

[17] J. H. Conway and N. J. A. Sloane, Sphere Packings, Lattices and Groups (Springer-Verlag, New York, 1988).

[18] O. R. Musin, Ann. Math. 168, 1 (2008).

[19] F. Pfender and G. M. Ziegler, Not. Am. Math. Soc. 51, 873 (2004).

[20] M. Skoge, A. Donev, F. H. Stillinger, and S. Torquato, Phys. Rev. E 74, 041127 (2006).

[21] M. Bishop and P. A. Whitlock, J. Chem. Phys. 123, 014507 (2005).

[22] R. J. Speedy, J. Phys.: Condens. Matter 10, 4387 (1998).

[23] J. P. J. Michels and N. J. Trappeniers, Phys. Lett. 104A, 425 (1984).

[24] W. B. Streett, H. J. Raveche, and R. D. Mountain, J. Chem. Phys. 61, 1960 (1974).

[25] J.-L. Colot and M. Baus, Phys. Lett. A 119, 135 (1986).

[26] D. Frenkel and B. Smit, Understanding Molecular Simulation (Academic, San Diego, 2002).

[27] D. Frenkel and A. J. C. Ladd, J. Chem. Phys. 81, 3188 (1984).

[28] P. R. ten Wolde, M. J. Ruiz-Montero, and D. Frenkel, J. Chem. Phys. 104, 9932 (1996).

[29] S. Auer and D. Frenkel, Nature (London) 409, 1020 (2001).

[30] S. Alexander and J. P. McTague, Phys. Rev. Lett. 41, 702 (1978).

[31] M. Volmer and A. Weber, Z. Phys. Chem. 119, 277 (1926). 